\title{
New refinements for integral and sum forms of Hölder inequality
}

İmdat Işcan ${ }^{1 *}$ (I)

${ }^{\text {*Correspondence: }}$

imdati@yahoo.com

'Department of Mathematics,

Faculty of Arts and Sciences,

Giresun University, Giresun, Turkey

\begin{abstract}
In this paper, we establish new refinements for integral and sum forms of Hölder inequality. Many existing inequalities related to the Hölder inequality can be improved via newly obtained inequalities, which we illustrate by an application.
\end{abstract}

MSC: Primary 26D15; secondary 26A51

Keywords: Hölder inequality; Young inequality; Integral inequalities;

Hermite-Hadamard-type inequality

\section{Introduction}

The famous Young inequality for two scalars is the $t$-weighted arithmetic-geometric mean inequality. This inequality says that if $u, v>0$ and $\theta \in[0,1]$, then

$$
u^{\theta} v^{1-\theta} \leq \theta u+(1-\theta) v
$$

with equality if and only if $u=v$. Let $r, s>1$ be such that $1 / r+1 / s=1$. Inequality (1) can be written as

$$
u v \leq \frac{u^{r}}{r}+\frac{v^{s}}{s}
$$

for $u, v \geq 0$. In this form, inequality (2) was used to prove the celebrated Hölder inequality, which is one of the most important inequalities of analysis. It contributes a wide area of pure and applied mathematics and plays a key role in resolving many problems in social, cultural science, and natural sciences.

Theorem 1.1 (Hölder inequality for integrals [7]) Let $p>1$ and $1 / p+1 / q=1$. If $\varphi$ and $\psi$ are real functions defined on $[u, v]$ such that $|\varphi|^{p}$ and $|\psi|^{q}$ are integrable functions on $[u, v]$, then

$$
\int_{u}^{v}|\varphi(\zeta) \psi(\zeta)| d \zeta \leq\left(\int_{u}^{v}|\varphi(\zeta)|^{p} d \zeta\right)^{1 / p}\left(\int_{u}^{v}|\psi(\zeta)|^{q} d \zeta\right)^{1 / q}
$$

with equality if and only if $A|\varphi(\zeta)|^{p}=B|\psi(\zeta)|^{q}$ almost everywhere for some constants $A$ and $B$.

(c) The Author(s) 2019. This article is distributed under the terms of the Creative Commons Attribution 4.0 International License (http://creativecommons.org/licenses/by/4.0/), which permits unrestricted use, distribution, and reproduction in any medium, provided you give appropriate credit to the original author(s) and the source, provide a link to the Creative Commons license, and indicate if changes were made. 
Theorem 1.2 (Hölder inequality for sums [7]) Let $u=\left(u_{1}, \ldots, u_{m}\right)$ and $v=\left(v_{1}, \ldots, v_{m}\right)$ be two positive $n$-tuples, and let $p, q>0$ be such that $1 / p+1 / q=1$. Then we have

$$
\sum_{n=1}^{m} u_{n} v_{n} \leq\left(\sum_{n=1}^{m} u_{n}^{p}\right)^{1 / p}\left(\sum_{n=1}^{m} v_{n}^{q}\right)^{1 / q}
$$

with equality (4) if and only if $u^{p}$ and $v^{q}$ are proportional.

Of course, the Hölder's inequality has been extensively explored and tested to a new situation by a number of scientists. Many its generalizations and refinements have been obtained so far. See, for example, [1, 2, 4-12] and the references therein. In this paper, using a simple proof method, we obtain some new refinements for integral and sum forms of Hölder's.

\section{Main results}

Theorem 2.1 Let $p>1$ and $1 / p+1 / q=1$. If $\varphi$ and $\psi$ are real functions defined on $[u, v]$ such that $|\varphi|^{p}$ and $|\psi|^{q}$ are integrable functions on $[u, v]$, then

(i)

$$
\begin{aligned}
\int_{u}^{v}|\varphi(\zeta) \psi(\zeta)| d \zeta \\
\quad \leq \frac{1}{v-u}\left\{\left(\int_{u}^{v}(v-\zeta)|\varphi(\zeta)|^{p} d \zeta\right)^{1 / p}\left(\int_{u}^{v}(v-\zeta)|\psi(\zeta)|^{q} d \zeta\right)^{1 / q}\right. \\
\left.\quad+\left(\int_{u}^{v}(\zeta-u)|\varphi(\zeta)|^{p} d \zeta\right)^{1 / p}\left(\int_{u}^{v}(\zeta-u)|\psi(\zeta)|^{q} d \zeta\right)^{1 / q}\right\}
\end{aligned}
$$

(ii)

$$
\begin{aligned}
& \frac{1}{v-u}\left\{\left(\int_{u}^{v}(v-\zeta)|\varphi(\zeta)|^{p} d \zeta\right)^{1 / p}\left(\int_{u}^{v}(v-\zeta)|\psi(\zeta)|^{q} d \zeta\right)^{1 / q}\right. \\
& \left.+\left(\int_{u}^{v}(\zeta-u)|\varphi(\zeta)|^{p} d \zeta\right)^{1 / p}\left(\int_{u}^{v}(\zeta-u)|\psi(\zeta)|^{q} d \zeta\right)^{1 / q}\right\} \\
& \leq\left(\int_{u}^{v}|\varphi(\zeta)|^{p} d \zeta\right)^{1 / p}\left(\int_{u}^{v}|\psi(\zeta)|^{q} d \zeta\right)^{1 / q}
\end{aligned}
$$

Proof (i) First (short) method: Using the Hölder inequality (3), we easily see that

$$
\begin{aligned}
\int_{u}^{v}|\varphi(\zeta) \psi(\zeta)| d \zeta \\
\quad=\frac{1}{v-u}\left\{\int_{u}^{v}\left|(v-\zeta)^{1 / p} \varphi(\zeta)(v-\zeta)^{1 / q} \psi(\zeta)\right| d \zeta\right. \\
\left.\quad+\int_{u}^{v}\left|(\zeta-u)^{1 / p} \varphi(\zeta)(\zeta-u)^{1 / q} \psi(\zeta)\right| d \zeta\right\} \\
\quad \leq \frac{1}{v-u}\left\{\left(\int_{u}^{v}(v-\zeta)|\varphi(\zeta)|^{p} d \zeta\right)^{1 / p}\left(\int_{u}^{v}(v-\zeta)|\psi(\zeta)|^{q} d \zeta\right)^{1 / q}\right.
\end{aligned}
$$


İşcan Journal of Inequalities and Applications

(2019) 2019:304

Page 3 of 11

$$
\left.+\left(\int_{u}^{v}(\zeta-u)|\varphi(\zeta)|^{p} d \zeta\right)^{1 / p}\left(\int_{u}^{v}(\zeta-u)|\psi(\zeta)|^{q} d \zeta\right)^{1 / q}\right\}
$$

Second (long) method: Let $\alpha \in[0,1]$. Applying (3) on the subinterval $[u, \alpha v+(1-\alpha) u]$ and on the subinterval $\alpha v+(1-\alpha) u, v]$, respectively, we get

$$
\int_{u}^{\alpha \nu+(1-\alpha) u}|\varphi(\zeta) \psi(\zeta)| d \zeta \leq\left(\int_{u}^{\alpha \nu+(1-\alpha) u}|\varphi(\zeta)|^{p} d \zeta\right)^{1 / p}\left(\int_{a}^{\lambda b+(1-\lambda) a}|\psi(\zeta)|^{q} d \zeta\right)^{1 / q}
$$

and

$$
\int_{\alpha \nu+(1-\alpha) u}^{v}|\varphi(\zeta) \psi(\zeta)| d \zeta \leq\left(\int_{\alpha \nu+(1-\alpha) u}^{v}|\varphi(\zeta)|^{p} d \zeta\right)^{1 / p}\left(\int_{\alpha \nu+(1-\alpha) u}^{v}|\psi(\zeta)|^{q} d \zeta\right)^{1 / q}
$$

Adding the resulting inequalities, we get

$$
\begin{aligned}
\int_{u}^{v}|\varphi(\zeta) \psi(\zeta)| d \zeta \leq & \left(\int_{u}^{\alpha \nu+(1-\alpha) u}|\varphi(\zeta)|^{p} d \zeta\right)^{1 / p}\left(\int_{a}^{\lambda b+(1-\lambda) a}|\psi(\zeta)|^{q} d \zeta\right)^{1 / q} \\
& +\left(\int_{\alpha \nu+(1-\alpha) u}^{v}|\varphi(\zeta)|^{p} d \zeta\right)^{1 / p}\left(\int_{\alpha \nu+(1-\alpha) u}^{v}|\psi(\zeta)|^{q} d \zeta\right)^{1 / q}
\end{aligned}
$$

By the change of variable $\zeta=\theta v+(1-\theta) u$ on the right-hand side integrals in (7), we have

$$
\begin{aligned}
& \int_{u}^{v}|\varphi(\zeta) \psi(\zeta)| d \zeta \\
& \quad \leq(v-u)\left\{\left(\int_{0}^{\theta}|\varphi(\theta v+(1-\theta) u)|^{p} d \theta\right)^{1 / p}\left(\int_{0}^{\theta}|\psi(\theta v+(1-\theta) u)|^{q} d \theta\right)^{1 / q}\right. \\
& \left.\quad+\left(\int_{\theta}^{1}|\varphi(\theta v+(1-\theta) u)|^{p} d \theta\right)^{1 / p}\left(\int_{\theta}^{1}|\psi(\theta v+(1-\theta) u)|^{q} d \theta\right)^{1 / q}\right\}
\end{aligned}
$$

Integrating both sides of this inequality over $[0,1]$ with respect to $\alpha$, we obtain that

$$
\begin{aligned}
& \int_{u}^{v}|\varphi(\zeta) \psi(\zeta)| d \zeta \\
& \quad \leq(v-u)\left\{\int_{0}^{1}\left(\int_{0}^{\theta}|\varphi(\theta v+(1-\theta) u)|^{p} d \theta\right)^{1 / p}\left(\int_{0}^{\theta}|\psi(\theta v+(1-\theta) u)|^{q} d \theta\right)^{1 / q} d \alpha\right. \\
& \left.\quad+\int_{0}^{1}\left(\int_{\theta}^{1}|\varphi(\theta v+(1-\theta) u)|^{p} d \theta\right)^{1 / p}\left(\int_{\theta}^{1}|\psi(\theta v+(1-\theta) u)|^{q} d \theta\right)^{1 / q} d \alpha\right\} .
\end{aligned}
$$

Then applying the Hölder inequality to the right-hand side integrals in the last inequality, we have

$$
\begin{aligned}
& \int_{u}^{v}|\varphi(\zeta) \psi(\zeta)| d \zeta \\
& \quad \leq(v-u)\left\{\left(\int_{0}^{1} \int_{0}^{\theta}|\varphi(\theta v+(1-\theta) u)|^{p} d \theta d \alpha\right)^{1 / p}\right. \\
& \quad \times\left(\int_{0}^{1} \int_{0}^{\theta}|\psi(\theta v+(1-\theta) u)|^{q} d \theta d \alpha\right)^{1 / q}
\end{aligned}
$$


scan Journal of Inequalities and Applications

(2019) 2019:304

Page 4 of 11

$$
\begin{aligned}
& +\left(\int_{0}^{1} \int_{\theta}^{1}|\varphi(\theta v+(1-\theta) u)|^{p} d \theta d \alpha\right)^{1 / p} \\
& \left.\times\left(\int_{0}^{1} \int_{\theta}^{1}|\psi(\theta v+(1-\theta) u)|^{q} d \theta d \alpha\right)^{1 / q}\right\} .
\end{aligned}
$$

By the Fubini theorem and the change of variable $\theta=(\zeta-u) /(v-u)$ we get

$$
\begin{aligned}
& \int_{u}^{v}|\varphi(\zeta) \psi(\zeta)| d \zeta \\
& \leq(v-u)\left\{\left(\int_{0}^{1} \int_{\theta}^{1}|\varphi(\theta v+(1-\theta) u)|^{p} d \alpha d \theta\right)^{1 / p}\right. \\
& \times\left(\int_{0}^{1} \int_{\theta}^{1}|\psi(\theta v+(1-\theta) u)|^{q} d \alpha d \theta\right)^{1 / q} \\
&+\left(\int_{0}^{1} \int_{0}^{\theta}|\varphi(\theta v+(1-\theta) u)|^{p} d \alpha d \theta\right)^{1 / p} \\
&\left.\times\left(\int_{0}^{1} \int_{0}^{\theta}|\psi(\theta v+(1-\theta) u)|^{q} d \alpha d \theta\right)^{1 / q}\right\} \\
&=(v-u)\left\{\left(\int_{0}^{1}(1-\theta)|\varphi(\theta v+(1-\theta) u)|^{p} d \theta\right)^{1 / p}\right. \\
& \times\left(\int_{0}^{1}(1-\theta)|\psi(\theta v+(1-\theta) u)|^{q} d \theta\right)^{1 / q} \\
&\left.+\left(\int_{0}^{1} \theta|\varphi(\theta v+(1-\theta) u)|^{p} d \theta\right)^{1 / p}\left(\int_{0}^{1} \theta|\psi(\theta v+(1-\theta) u)|^{q} d \theta\right)^{1 / q}\right\} \\
&= 1 \\
&\left.+\left(\int_{u}^{v}(\zeta-u)|\varphi(\zeta)|^{p} d \zeta\right)^{1 / p}\left(\int_{u}^{v}(\zeta-u)|\psi(\zeta)|^{q} d \zeta\right)^{1 / q}\right\} \\
& \\
&\left.=(v-\zeta)|\varphi(\zeta)|^{p} d \zeta\right)^{1 / p}\left(\int_{u}^{v}(v-\zeta)|\psi(\zeta)|^{q} d \zeta\right)^{1 / q}
\end{aligned}
$$

(ii) First, let us consider the case

$$
\left(\int_{u}^{v}|\varphi(\zeta)|^{p} d \zeta\right)^{1 / p}\left(\int_{u}^{v}|\psi(\zeta)|^{q} d \zeta\right)^{1 / q}=0
$$

Then, $\varphi(\zeta)=0$ for almost every $\zeta \in[u, v]$ or $\psi(\zeta)=0$ for almost everywhere $\zeta \in[u, v]$. Thus we have

$$
\int_{u}^{v}|\varphi(\zeta) \psi(\zeta)| d \zeta=0
$$

Therefore inequality (6) is trivial in this case.

Finally, we consider the case

$$
I=\left(\int_{u}^{v}|\varphi(\zeta)|^{p} d \zeta\right)^{1 / p}\left(\int_{u}^{v}|\psi(\zeta)|^{q} d \zeta\right)^{1 / q} \neq 0 .
$$


Then

$$
\begin{aligned}
& \frac{1}{(v-u) I}\left\{\left(\int_{u}^{v}(v-\zeta)|\varphi(\zeta)|^{p} d \zeta\right)^{1 / p}\left(\int_{u}^{v}(v-\zeta)|\psi(\zeta)|^{q} d \zeta\right)^{1 / q}\right. \\
& \left.+\left(\int_{u}^{v}(\zeta-u)|\varphi(\zeta)|^{p} d \zeta\right)^{1 / p}\left(\int_{u}^{v}(\zeta-u)|\psi(\zeta)|^{q} d \zeta\right)^{1 / q}\right\} \\
& \leq \frac{1}{v-u}\left\{\left(\frac{\int_{u}^{v}(v-\zeta)|\varphi(\zeta)|^{p} d \zeta}{\int_{u}^{v}|\varphi(\zeta)|^{p} d \zeta}\right)^{1 / p}\left(\frac{\int_{u}^{v}(v-\zeta)|\psi(\zeta)|^{q} d \zeta}{\int_{u}^{v}|\psi(\zeta)|^{q} d \zeta}\right)^{1 / q}\right. \\
& \left.+\left(\frac{\int_{u}^{v}(\zeta-u)|\varphi(\zeta)|^{p} d \zeta}{\int_{u}^{v}|\varphi(\zeta)|^{p} d \zeta}\right)^{1 / p}\left(\frac{\int_{u}^{v}(\zeta-u)|\psi(\zeta)|^{q} d \zeta}{\int_{u}^{v}|\psi(\zeta)|^{q} d \zeta}\right)^{1 / q}\right\}
\end{aligned}
$$

Applying (1) to the right-hand side integrals of the last inequality, we get

$$
\begin{aligned}
& \frac{1}{(v-u) I}\left\{\left(\int_{u}^{v}(v-\zeta)|\varphi(\zeta)|^{p} d \zeta\right)^{1 / p}\left(\int_{u}^{v}(v-\zeta)|\psi(\zeta)|^{q} d \zeta\right)^{1 / q}\right. \\
& \left.+\left(\int_{u}^{v}(\zeta-u)|\varphi(\zeta)|^{p} d \zeta\right)^{1 / p}\left(\int_{u}^{v}(\zeta-u)|\psi(\zeta)|^{q} d \zeta\right)^{1 / q}\right\} \\
& \leq \frac{1}{v-u}\left\{\frac{\int_{u}^{v}(v-\zeta)|\varphi(\zeta)|^{p} d \zeta}{p \int_{u}^{v}|\varphi(\zeta)|^{p} d \zeta}+\frac{\int_{u}^{v}(v-\zeta)|\psi(\zeta)|^{q} d \zeta}{q \int_{u}^{v}|\psi(\zeta)|^{q} d \zeta}\right. \\
& \left.\quad+\frac{\int_{u}^{v}(\zeta-u)|\varphi(\zeta)|^{p} d \zeta}{p \int_{u}^{v}|\varphi(\zeta)|^{p} d \zeta}+\frac{\int_{u}^{v}(\zeta-u)|\psi(\zeta)|^{q} d \zeta}{q \int_{u}^{v}|\psi(\zeta)|^{q} d \zeta}\right\} \\
& =\frac{1}{p}+\frac{1}{q}=1
\end{aligned}
$$

This completes the proof.

Remark 2.1 Inequality (6) shows that inequality (5) is better than inequality (3).

More general versions of Theorem 2.1 are given in the following:

Theorem 2.2 Let $p>1$ and $1 / p+1 / q=1$. If $\varphi$ and $\psi$ are real functions defined on $[u, v]$ such that $|\varphi|^{p}$ and $|\psi|^{q}$ are integrable functions on $[u, v]$, then

(i)

$$
\begin{aligned}
& \int_{u}^{v}|\varphi(\zeta) \psi(\zeta)| d \zeta \\
& \quad \leq\left\{\left(\int_{u}^{v} \alpha(\zeta)|\varphi(\zeta)|^{p} d \zeta\right)^{1 / p}\left(\int_{u}^{v} \alpha(\zeta)|\psi(\zeta)|^{q} d \zeta\right)^{1 / q}\right. \\
& \left.\quad+\left(\int_{u}^{v} \beta(\zeta)|\varphi(\zeta)|^{p} d \zeta\right)^{1 / p}\left(\int_{u}^{v} \beta(\zeta)|\psi(\zeta)|^{q} d \zeta\right)^{1 / q}\right\}
\end{aligned}
$$

where $\alpha, \beta:[u, v] \rightarrow[0, \infty)$ are continuous functions such that $\alpha(\zeta)+\beta(\zeta)=1, \zeta \in[u, v]$

(ii)

$$
\int_{u}^{v}|\varphi(\zeta) \psi(\zeta)| d \zeta
$$




$$
\leq \sum_{i=1}^{n}\left(\int_{a}^{b} \alpha_{i}(\zeta)|\varphi(\zeta)|^{p} d \zeta\right)^{1 / p}\left(\int_{a}^{b} \alpha_{i}(\zeta)|\psi(\zeta)|^{q} d \zeta\right)^{1 / q}
$$

where $\alpha_{i}:[u, v] \rightarrow[0, \infty), i=1,2, \ldots, n$, are continuous functions such that $\sum_{i=1}^{n} \alpha_{i}(\zeta)=1, \zeta \in[u, v]$.

Proof The proof of the theorem is easily seen by using a similar method as in the proof of Theorem 2.1.

Remark 2.2 We easily see that the inequalities obtained in Theorem 2.2 are better than inequality (3).

Remark 2.3 (i) Taking $\alpha(\zeta)=\sin ^{2} \zeta$ and $\beta(\zeta)=\cos ^{2} \zeta$ in inequality (8), we have

$$
\begin{aligned}
& \int_{u}^{v}|\varphi(\zeta) \psi(\zeta)| d \zeta \\
& \quad \leq\left\{\left(\int_{u}^{v} \sin ^{2} \zeta|\varphi(\zeta)|^{p} d \zeta\right)^{1 / p}\left(\int_{u}^{v} \sin ^{2} \zeta|\psi(\zeta)|^{q} d \zeta\right)^{1 / q}\right. \\
& \left.\quad+\left(\int_{u}^{v} \cos ^{2} \zeta|\varphi(\zeta)|^{p} d \zeta\right)^{1 / p}\left(\int_{u}^{v} \cos ^{2} \zeta|\psi(\zeta)|^{q} d \zeta\right)^{1 / q}\right\}
\end{aligned}
$$

(ii) Taking $\alpha(\zeta)=\frac{v-\zeta}{v-u}$ and $\beta(\zeta)=\frac{\zeta-u}{v-u}$ in inequality (8), we have inequality (5).

Theorem 2.3 Let $u=\left(u_{1}, \ldots, u_{m}\right)$ and $v=\left(v_{1}, \ldots, v_{m}\right)$ be two positive $n$-tuples, and let $p, q>$ 0 be such that $1 / p+1 / q=1$. Then

(i)

$$
\begin{aligned}
\sum_{n=1}^{m} u_{n} v_{n} \leq & \frac{1}{m}\left\{\left(\sum_{n=1}^{m} n u_{n}^{p}\right)^{1 / p}\left(\sum_{n=1}^{m} n v_{n}^{q}\right)^{1 / q}\right. \\
& \left.+\left(\sum_{n=1}^{m}(m-n) u_{n}^{p}\right)^{1 / p}\left(\sum_{n=1}^{m}(m-n) v_{n}^{q}\right)^{1 / q}\right\} ;
\end{aligned}
$$

(ii)

$$
\begin{aligned}
& \frac{1}{m}\left\{\left(\sum_{n=1}^{m} n u_{n}^{p}\right)^{1 / p}\left(\sum_{n=1}^{m} n v_{n}^{q}\right)^{1 / q}+\left(\sum_{n=1}^{m}(m-n) u_{n}^{p}\right)^{1 / p}\left(\sum_{n=1}^{m}(m-n) v_{n}^{q}\right)^{1 / q}\right\} \\
& \quad \leq\left(\sum_{n=1}^{m} u_{n}^{p}\right)^{1 / p}\left(\sum_{n=1}^{m} v_{n}^{q}\right)^{1 / q} .
\end{aligned}
$$

Proof (i) Using the Hölder inequality in (4), we easily see that

$$
\begin{aligned}
& \sum_{n=1}^{m} u_{n} v_{n} \\
& \quad=\sum_{n=1}^{m}\left(\frac{n}{m}+\frac{m-n}{m}\right) u_{n} v_{n}
\end{aligned}
$$




$$
\begin{aligned}
= & \frac{1}{m}\left\{\sum_{n=1}^{m} n^{1 / p} u_{n} n^{1 / q} v_{n}+\sum_{n=1}^{m}(m-n)^{1 / p} u_{n}(m-n)^{1 / q} v_{n}\right\} \\
\leq & \frac{1}{m}\left\{\left(\sum_{n=1}^{m} n u_{n}^{p}\right)^{1 / p}\left(\sum_{n=1}^{m} n v_{n}^{q}\right)^{1 / q}\right. \\
& \left.+\left(\sum_{n=1}^{m}(m-n) u_{n}^{p}\right)^{1 / p}\left(\sum_{n=1}^{m}(m-n) v_{n}^{q}\right)^{1 / q}\right\} .
\end{aligned}
$$

(ii) Let us first consider the case

$$
\left(\sum_{n=1}^{m} u_{n}^{p}\right)^{1 / p}\left(\sum_{n=1}^{m} v_{n}^{q}\right)^{1 / q}=0 .
$$

Then $u_{n}=0$ for $n=1,2, \ldots, m$ or $v_{n}=0$ for $n=1,2, \ldots, m$. Thus we have

$$
\sum_{n=1}^{m} u_{n} v_{n}=0
$$

Therefore inequality (10) is trivial in this case.

Finally, we consider the case

$$
S=\left(\sum_{n=1}^{m} u_{n}^{p}\right)^{1 / p}\left(\sum_{n=1}^{m} v_{n}^{q}\right)^{1 / q} \neq 0 .
$$

Then

$$
\begin{aligned}
\frac{1}{m S}\left\{\left(\sum_{n=1}^{m} n u_{n}^{p}\right)^{1 / p}\left(\sum_{n=1}^{m} n v_{n}^{q}\right)^{1 / q}+\left(\sum_{n=1}^{m}(m-n) u_{n}^{p}\right)^{1 / p}\left(\sum_{n=1}^{m}(m-n) v_{n}^{q}\right)^{1 / q}\right\} \\
=\frac{1}{m}\left\{\left(\frac{\sum_{n=1}^{m} n u_{n}^{p}}{\sum_{n=1}^{m} u_{n}^{p}}\right)^{1 / p}\left(\frac{\sum_{n=1}^{m} n v_{n}^{q}}{\sum_{n=1}^{m} v_{n}^{q}}\right)^{1 / q}\right. \\
\left.+\left(\frac{\sum_{n=1}^{m}(m-n) u_{n}^{p}}{\sum_{n=1}^{m} u_{n}^{p}}\right)^{1 / p}\left(\frac{\sum_{n=1}^{m}(m-n) v_{n}^{q}}{\sum_{n=1}^{m} v_{n}^{q}}\right)^{1 / q}\right\} .
\end{aligned}
$$

Applying (1) to the right-hand side sums of the last inequality, we get

$$
\begin{aligned}
& \frac{1}{m S}\left\{\left(\sum_{n=1}^{m} n u_{n}^{p}\right)^{1 / p}\left(\sum_{n=1}^{m} n v_{n}^{q}\right)^{1 / q}+\left(\sum_{n=1}^{m}(m-n) u_{n}^{p}\right)^{1 / p}\left(\sum_{n=1}^{m}(m-n) v_{n}^{q}\right)^{1 / q}\right\} \\
& \quad \leq \frac{1}{m}\left\{\frac{\sum_{n=1}^{m} n u_{n}^{p}}{p \sum_{n=1}^{m} u_{n}^{p}}+\frac{\sum_{n=1}^{m} n v_{n}^{q}}{q \sum_{n=1}^{m} v_{n}^{q}}+\frac{\sum_{n=1}^{m}(m-n) u_{n}^{p}}{p \sum_{n=1}^{m} u_{n}^{p}}+\frac{\sum_{n=1}^{m}(m-n) v_{n}^{q}}{q \sum_{n=1}^{m} v_{n}^{q}}\right\} \\
& \quad=\frac{1}{p}+\frac{1}{q}=1 .
\end{aligned}
$$

This completes the proof.

Remark 2.4 Inequality (10) shows that inequality (9) is better than inequality (4). 
More general versions of Theorem 2.1 are given in the following:

Theorem 2.4 Let $u=\left(u_{1}, \ldots, u_{m}\right)$ and $v=\left(v_{1}, \ldots, v_{m}\right)$ be two positive $n$-tuples, and let $p, q>$ 0 be such that $1 / p+1 / q=1$.

(i) Let $c=\left(c_{1}, \ldots, c_{m}\right)$ and $d=\left(d_{1}, \ldots, d_{m}\right)$ be two positive $n$-tuples such that $c_{n}+d_{n}=1, n=1,2, \ldots, m$. Then

$$
\begin{aligned}
\sum_{n=1}^{m} u_{n} v_{n} \leq & \left\{\left(\sum_{n=1}^{m} c_{n} u_{n}^{p}\right)^{1 / p}\left(\sum_{n=1}^{m} c_{n} v_{n}^{q}\right)^{1 / q}\right. \\
& \left.+\left(\sum_{n=1}^{m} d_{n} u_{n}^{p}\right)^{1 / p}\left(\sum_{n=1}^{m} d_{n} v_{n}^{q}\right)^{1 / q}\right\} .
\end{aligned}
$$

(ii) Let $c^{(i)}=\left(c_{1}^{(i)}, \ldots, c_{m}^{(i)}\right), i=1,2, \ldots, k$, be positive $n$-tuples such that $\sum_{i=1}^{k} c_{n}^{(i)}=1, n=1,2, \ldots, m$. Then

$$
\sum_{n=1}^{m} u_{n} v_{n} \leq \sum_{i=1}^{k}\left\{\left(\sum_{n=1}^{m} c_{n}^{(i)} u_{n}^{p}\right)^{1 / p}\left(\sum_{n=1}^{m} c_{n}^{(i)} v_{n}^{q}\right)^{1 / q}\right\}
$$

Proof The proof of the theorem is easily seen by using a similar method as in the proof of Theorem 2.1.

Remark 2.5 We easily see that the inequalities obtained in Theorem 2.4 are better than inequality (4).

Remark 2.6 (i) Taking $c=\left(\sin ^{2} 1, \ldots, \sin ^{2} m\right)$ and $d=\left(\cos ^{2} 1, \ldots, \cos ^{2} m\right)$ in inequality (11) of Theorem 2.4 , we have

$$
\begin{aligned}
\sum_{n=1}^{m} u_{n} v_{n} \leq & \left\{\left(\sum_{n=1}^{m} \sin ^{2} n u_{n}^{p}\right)^{1 / p}\left(\sum_{n=1}^{m} \sin ^{2} n v_{n}^{q}\right)^{1 / q}\right. \\
& \left.+\left(\sum_{n=1}^{m} \cos ^{2} n u_{n}^{p}\right)^{1 / p}\left(\sum_{n=1}^{m} \cos ^{2} n v_{n}^{q}\right)^{1 / q}\right\}
\end{aligned}
$$

(ii) Taking $c=\left(\frac{1}{m}, \frac{2}{m}, \ldots, 1\right)$ and $d=\left(\frac{m-1}{m}, \frac{m-2}{m}, \ldots, 0\right)$ in inequality (11) of Theorem 2.4, we have inequality (9).

\section{An application}

To obtain our main results, we need the following lemma of Dragomir et al. [3].

Lemma 3.1 Let $f: I^{\circ} \subseteq \mathbb{R} \rightarrow \mathbb{R}$ be a differentiable mapping on $I^{\circ}$, let $a, b \in I^{\circ}$ with $a<b$, and let $q>1$. Iff $\in L[a, b]$, then

$$
\frac{f(a)+f(b)}{2}-\frac{1}{b-a} \int_{a}^{b} f(x) d x=\frac{b-a}{2} \int_{0}^{1}(1-2 t) f^{\prime}(t a+(1-t) b) d t .
$$

Using this equality and the Hölder integral inequality, Dragomir et al. obtained the following inequality. 
Theorem 3.1 Let $f: I^{\circ} \subseteq \mathbb{R} \rightarrow \mathbb{R}$ be a differentiable mapping on $I^{\circ}$, and let $a, b \in I^{\circ}$ with $a<b$. If the new mapping $\left|f^{\prime}\right|^{q}$ is convex on $[a, b]$, then

$$
\left|\frac{f(a)+f(b)}{2}-\frac{1}{b-a} \int_{a}^{b} f(x) d x\right| \leq \frac{b-a}{2(p+1)^{1 / p}}\left[\frac{\left|f^{\prime}(a)\right|^{q}+\left|f^{\prime}(b)\right|^{q}}{2}\right]^{1 / q},
$$

where $1 / p+1 / q=1$.

If Theorem 3.1 is resulted again by using inequality (5) in Theorem 2.1, then we get the following result.

Theorem 3.2 Let $f: I^{\circ} \subseteq \mathbb{R} \rightarrow \mathbb{R}$ be a differentiable mapping on $I^{\circ}$, and let $a, b \in I^{\circ}$ with $a<b$. If the mapping $\left|f^{\prime}\right|^{q}$ is convex on $[a, b]$, then

$$
\begin{aligned}
& \left|\frac{f(a)+f(b)}{2}-\frac{1}{b-a} \int_{a}^{b} f(x) d x\right| \\
& \quad \leq \frac{b-a}{4(p+1)^{1 / p}}\left\{\left[\frac{2\left|f^{\prime}(a)\right|^{q}+\left|f^{\prime}(b)\right|^{q}}{3}\right]^{1 / q}+\left[\frac{\left|f^{\prime}(a)\right|^{q}+2\left|f^{\prime}(b)\right|^{q}}{3}\right]^{1 / q}\right\}
\end{aligned}
$$

where $1 / p+1 / q=1$.

Proof Using Lemma 3.1 and inequality (5), we find

$$
\begin{aligned}
& \left|\frac{f(a)+f(b)}{2}-\frac{1}{b-a} \int_{a}^{b} f(x) d x\right| \\
& \leq \frac{b-a}{2} \int_{0}^{1}|1-2 t|\left|f^{\prime}(t a+(1-t) b)\right| d t \\
& \leq \frac{b-a}{2}\left\{\left(\int_{0}^{1}(1-t)|1-2 t|^{p} d t\right)^{1 / p}\left(\int_{0}^{1}(1-t)\left|f^{\prime}(t a+(1-t) b)\right|^{q} d t\right)^{1 / q}\right. \\
& \left.\quad+\left(\int_{0}^{1} t|1-2 t|^{p} d t\right)^{1 / p}\left(\int_{0}^{1} t\left|f^{\prime}(t a+(1-t) b)\right|^{q} d t\right)^{1 / q}\right\} .
\end{aligned}
$$

Using the convexity of $\left|f^{\prime}\right|^{q}$, we have

$$
\begin{aligned}
\int_{0}^{1} t\left|f^{\prime}(t a+(1-t) b)\right|^{q} d t & \leq \int_{0}^{1} t\left[t\left|f^{\prime}(a)\right|^{q}+(1-t)\left|f^{\prime}(b)\right|^{q}\right] d t \\
& =\frac{2\left|f^{\prime}(a)\right|^{q}+\left|f^{\prime}(b)\right|^{q}}{6}
\end{aligned}
$$

and

$$
\begin{aligned}
\int_{0}^{1}(1-t)\left|f^{\prime}(t a+(1-t) b)\right|^{q} d t & =\int_{0}^{1} t\left|f^{\prime}(t b+(1-t) a)\right|^{q} d t \\
& \leq \frac{\left|f^{\prime}(a)\right|^{q}+2\left|f^{\prime}(b)\right|^{q}}{6}
\end{aligned}
$$

Further, since

$$
\int_{0}^{1} t|1-2 t|^{p} d t=\int_{0}^{1}(1-t)|1-2 t|^{p} d t
$$




$$
=\frac{1}{2(p+1)}
$$

a combination of (14)-(16) immediately gives the required inequality (13).

Remark 3.1 Since $\mu:[0, \infty) \rightarrow \mathbb{R}, \mu(x)=x^{\alpha}, 0<\alpha \leq 1$, is a concave function, for all $z, t \geq 0$, we have

$$
\mu\left(\frac{z+t}{2}\right)=\left(\frac{z+t}{2}\right)^{\alpha} \geq \frac{\mu(z)+\mu(t)}{2}=\frac{z^{\alpha}+t^{\alpha}}{2}
$$

from which we get

$$
\frac{1}{2}\left[\frac{2\left|f^{\prime}(a)\right|^{q}+\left|f^{\prime}(b)\right|^{q}}{3}\right]^{1 / q}+\frac{1}{2}\left[\frac{\left|f^{\prime}(a)\right|^{q}+2\left|f^{\prime}(b)\right|^{q}}{3}\right]^{1 / q} \leq\left[\frac{\left|f^{\prime}(a)\right|^{q}+\left|f^{\prime}(b)\right|^{q}}{2}\right]^{1 / q} .
$$

Thus we obtain

$$
\begin{aligned}
& \frac{b-a}{4(p+1)^{1 / p}}\left\{\left[\frac{2\left|f^{\prime}(a)\right|^{q}+\left|f^{\prime}(b)\right|^{q}}{3}\right]^{1 / q}+\left[\frac{\left|f^{\prime}(a)\right|^{q}+2\left|f^{\prime}(b)\right|^{q}}{3}\right]^{1 / q}\right\} \\
& \leq \frac{b-a}{2(p+1)^{1 / p}}\left[\frac{\left|f^{\prime}(a)\right|^{q}+\left|f^{\prime}(b)\right|^{q}}{2}\right]^{1 / q},
\end{aligned}
$$

which shows that inequality (13) is better than inequality (12).

\section{Conclusion}

In this paper, using a simple proof method, we obtained some new refinements for integral and sum forms of Hölder's inequality. Many existing inequalities related to the Hölder inequality can be improved via newly obtained inequalities, which we illustrate by an application. Moreover, our results can be recognized as significant methods in the fields of mathematics.

Acknowledgements

Not applicable.

Funding

There is no funding for this work.

Abbreviations

Not applicable.

Availability of data and materials

Not applicable.

Competing interests

The author declares that he has no competing interests.

Authors' contributions

The author read and approved the final manuscript.

\section{Publisher's Note}

Springer Nature remains neutral with regard to jurisdictional claims in published maps and institutional affiliations. 
References

1. Agahi, H., Yadollahzadeh, M.: Refinements of Hölder's and Minkowski's type inequalities for $\sigma-\oplus$-measures and pseudo-expectation. Soft Comput. 22, 8051-8058 (2018)

2. Beckenbach, E.F., Bellman, R. (eds.): Inequalities. Springer, Berlin (1961)

3. Dragomir, S.S., Agarwal, R.P.: Two inequalities for differentiable mappings and applications to special means of real numbers and to trapezoidal formula. Appl. Math. Lett. 11(5), 91-95 (1998)

4. Hardy, G.H., Littlewood, J.E., Polya, G.: Inequalities. Cambridge University Press, Cambridge (1952)

5. Kim, Y.I., Yang, X.: Generalizations and refinements of Hölder's inequality. Appl. Math. Lett. 25, 1094-1097 (2012)

6. Kwon, E.G., Bae, J.E.: On a refined Hölder's inequality. J. Math. Inequal. 10(1), 261-268 (2016)

7. Mitrinović, D.S., Pečarić, J.E., Fink, A.M.: Classical and New Inequalities in Analysis. Kluwer Academic, Dordrecht (1993)

8. Qiang, H., Hu, Z.: Generalizations of Hölder's and some related inequalities. Comput. Math. Appl. 61, 511-525 (2011)

9. Tian, J.F., Ha, M.H.: Properties of generalized sharp Hölder's inequalities. J. Math. Inequal. 11(2), $261-268$ (2017)

10. Tian, J.F., Ha, M.H.: Extensions of Hölder's inequality via pseudo-integral. Math. Probl. Eng. 2018, Article ID 4080619 (2018)

11. Tian, J.F., Ha, M.H., Wang, C.: Improvements of generalized Hölder's inequalities and their applications. J. Math. Inequal. 12(2), 459-471 (2018)

12. Wu, S.H.: Generalization of a sharp Hölder's inequality and its application. J. Math. Anal. Appl. 332, 741-750 (2007)

\section{Submit your manuscript to a SpringerOpen ${ }^{\circ}$ journal and benefit from:}

- Convenient online submission

Rigorous peer review

Open access: articles freely available online

High visibility within the field

- Retaining the copyright to your article

Submit your next manuscript at $\gg$ springeropen.com 